九州大学学術情報リポジトリ

Kyushu University Institutional Repository

\title{
Numerical Modeling of Low Level Horizontal Penetration Seeding of Supercooled Cloud with Liquid Carbon Dioxide
}

Javanmard, Sohai la

Laboratory of Agricultural Meteorology, Faculty of Agriculture, Kyushu University

Fukuta, Norihiko

Department of Meteorology, University of Utah

Nish iyama, Kouj i

Faculty of Engineering, Kyushu University

Suzuki, Yoshinori

Laboratory of Agricultural Meteorology, Faculty of Agriculture, Kyushu University

他

https://doi.org/10.5109/24269

出版情報：九州大学大学院農学研究院紀要. 43 (1/2)，pp. 239-255，1998-11. Kyushu University バージョン：

権利関係: 


\title{
Numerical Modeling of Low Level Horizontal Penetration Seeding of Supercooled Cloud with Liquid Carbon Dioxide
}

\author{
Sohaila Javanmard, Norihiko Fukuta*1, Kouji Nishiyama*2, \\ Yoshinori Suzuki, and Kenji Wakimizu
}

\author{
Laboratory of Agricultural Meteorology, Faculty of Agriculture, Kyushu University, \\ 6-10-1 Hakozaki, Higashi-ku, Fukuoka 812-8581, Japan \\ (Received July 25, 1998 and accepted August 7, 1998)
}

\begin{abstract}
Numerical modeling of low level horizontal penetration seeding of supercooled cloud using liquid carbon dioxide (Fukuta 1996) has been carried out for the first time. The model is timedependent, two-dimensional, covering a region $4 \mathrm{~km} \times 6 \mathrm{~km}$ in the $x$ and $z$ directions with $50 \mathrm{~m}$ grid intervals. We assume a supercooled cumulus clond has already been formed and the liquid water content as constant. with height. The motion of ice crystal thermal in cloud is assumed like that of cumulus cell according to Scorer (1972). The ice crystals are assumed to be spherical shape and grow by consuming supercooled cloud entrained into the ice thermal.

The model shows Roll up Expansion of the Twin Horizontal Ice crystal Thermal (RETHIT) due to buoyancy. Rise of horizontal ice crystal thermal while rotating, expanding and cntraining the supercooled cloud volume leads to formation of ice crystals which possess sufficiently large size and necessary fall velocity in a large cloud volume within a likely clout lifetime at the cloud top. Crcation of large crystals with fast fall velocity assures subscquent changes for effective precipitation development. The centrifugal force is also found to act on ice particles and help them spread. Optimal seeding rate of liquid carbon dioxide is assessed as about $10 \mathrm{gram}$ per second.
\end{abstract}

\section{INTRODUCTION}

Currently several methods are used for modification of supercooled clouds. Among these is the generation of ice crystals in the supercooled clouds with two mechanisms of homogeneous and heterogeneous ice nucleations. Ice nucleants most frequently used are silver iodide (AgI) (Vonnegut 1947) and dry ice (Schaefer 1946). A review on AgI and dry ice seeding problems is as follows:

The serious problem of AgI seeding, apart from details of ice nucleation mechanisms (Schaller and Fukuta 1979), is the strong temperature dependence in the number of active nuclei. The number increases many orders of magnitude when the temperature lowers. This leads to the problem of nucleating too many small ice crystals at low temperatures-high altitudes if the number were adjusted at high temperatures-low altitudes. As a result, ice crystals do not grow effectively to desirable sizes due to competition for available moisture. On the other hand, if the number were adjusted at a high altitude, too few ice crystals form and grow before reaching the upper portion of the cloud in the updraft to cause significant precipitation.

A method to drop dry ice pellets from above a supercooled cloud results in rapid upward movement of the generated ice thermal due to the vertically integration of the

*: Department of Mcteorology, Lniversity of Utah, Salt Lake City, Utah 84112, U.S.A.

*2 Faculty of Engineering; Kyushu University, 6-10-1 Hakozaki, Higashi-ku1, Fukuoka 812-8581, Japan. 
buoyant force caused by the phase change heat and reduces the number of ice crystals formed at low altitudes as well as increases those at higher altitudes. The time for the diffusion of the ice thermal and therefore ice crystal growth is too short. The thermal of small ice crystals rises rapidly to the cloud top, stabilizes there due to the added buoyancy and their small fall velocity, and cannot be effectively involved in precipitation formation process within the cloud lifetime.

In order to achieve effective modification of supercooled clouds, Fukuta (1996a and b) formulated a new seeding method which can not only avoid the above mentionted problems but also maximize the seeding reactions involved. For improving the cloud reactions, the basic proccsses in cloud microphysics and dynamics are examined. It was realized that the ice crystal growth requirements, i.e. the time and co-existence of supercooled droplets which are to be brought in by entrainment, have to be satisfied to the fullest exteni. A method which satisfies the above conditions is expected to work with supercooled clouds, which consist of small water droplets at temperatures below freezing. It causes Roll up Expansion of Twin Horizontal Ice crystal Thermals (RETHIT) followed by Falling-growth Induced Lateral Air Spreading (FILAS) (Fukuta 1998), with a low level horizontal penetration sceding of liquid carbon dioxide (LC), and it has already been developed and tested in supercooled stratus clouds and fogs with marked results (Fukuta 1996b). This paper reports the results of our first effort to numerically simulate the detailed processes of a new method of LC secding specifically focusing on microphysics and dynamics interactions of RETHIT and to assess the optimal seeding rate.

\section{NUMERICAL MODEL}

\section{Microphysical processes}

a. Ice nucleation

The first process which takes place in the cloud seeding is ice nucleation. There exist two main ice nucleation mechanisms, homogeneous and heterogeneous ice nucleations as mentioned above. Homogeneous ice nucleation generates ice crystals by strong cooling such as dry ice (Fukutia el al. 1971; Fukuta 1987) in which the number of generated ice crystals by the coolant is nearly independent of the temperature. Whereas, hetcrogeneous ice nucleation, typically represented by silver iodide (AgI), is strongly temperature dependent (Garvey 1975). Organic ice nucleants such as metaldehyde (Fukuta 1963) and 1.5-dihydroxynaphthalence (Fukuta 1966) show a lesser degree of temperature dependence in numbers (Plooster and Fukuta 1975). The number of active AgI particles increases 4 orders of magnitude as the temperature drops from -6 to $-10^{\circ} \mathrm{C}$. The temperature dependent activity of the ice nucleant leads to an insufficient number of ice crystal formations at a low altitude or under slight supercooling if the number is adjusted to a high altitude. On the other hand, at a high altitude or under large supercooling, the large number of ice nucleations leads to formation of small crystals that do not fall well if the number is adjusted to a low altitude.

The number of ice crystal generations per gram of LC is basically the same as that of dry ice and is probably close to the maximum the homogeneous mechanism can achieve (Fukuta 1988). Consequently, due to its temperature independent ice nucleating activity 
and other practical factors which will be described in the following, LC is chosen.

\section{b. Properties of liquid $\mathrm{CO}_{2}$}

Some practical properties of LC have been presented by Fukuta (1996a). They are as follows;

(i ) Low price (nearly $1 / 2$ of dry ice).

(ii) Non-toxic and safe to the environment (Cooper and Jolly 1970; Klein 1978).

(iii) Non-flammable and produces no asphyxiating fumes in the cockpit unike dry ice.

(iv) Convenient for long term storage.

(v) A high pressure liquid for continuous and accurate discharging with its own vapor pressure $\left(52\right.$ atm at $\left.15^{\circ} \mathrm{C}\right)$.

(vi) LC has high ice nucleation efficiency due to strong cooling (Fukuta 1965) and suitable characteristics for horizontal penetration seeding at low altitude.

\section{c. Intensity of ice crystal source}

At the beginning of seeding, the ice thermal is in the form of a horizontal line. The total number of ice crystals formed per $1 \mathrm{~m}$ of flight path which is called the intensity of the ice crystal line source $\left(N_{T}\right)$ may be determined as follows:

Using the number of ice crystals generated by LC which is independent of temperature, $n=10^{13} \mathrm{~g}^{-1}$ and the seeding rate, $P=10 \mathrm{~g} / \mathrm{s}$ and the speed of aircraft, $u=100 \mathrm{~m} / \mathrm{s}$, the intensity of ice crystal line source gives $N_{T}=\frac{n P}{u}-=10^{12} \mathrm{~m}^{-1}$

\section{d. Ice crystal growth}

It is assumed that supercooled cloud has been already formed and liquit water content of the cloud is assumed as constant with height. Ice crystals generated by the seeding grow by consuming supercooled cloud entrained into the ice thermal. The ice crystals are assumed to be in a spherical shape with the density, $\rho_{i}=0.917 \mathrm{~g} / \mathrm{cm}^{3}$, and uniform in size. The increase in mass of ice crystals in the time interval $5 \mathrm{~s}$ is expressed as

$$
\Delta m_{i}=\Delta V \frac{W}{N}_{T_{T}}
$$

where $W_{L}=0.5 \mathrm{~g} / \mathrm{m}^{3}$ is liquid water content of supercooled cloud, $\Delta V$ the increment in the volume of thermal in the time interval, $\Delta \mathrm{t}=5 \mathrm{~s}$. Then, $\Delta V$ may be obtained as

$$
\mathrm{DV}=\left(\pi R^{12}-\pi R^{2}\right) \times l,
$$

where $\mathrm{R}^{\prime}$ is the radius of one of the twin cylinders of thermal at the time $t$ as shown in Fig. $1, \mathrm{R}$ the radius at previous time step $(\mathrm{t}-\Delta \mathrm{t})$ and $l$ the length of the cylinder along the flight path which is taken as $1 \mathrm{~m}$. 


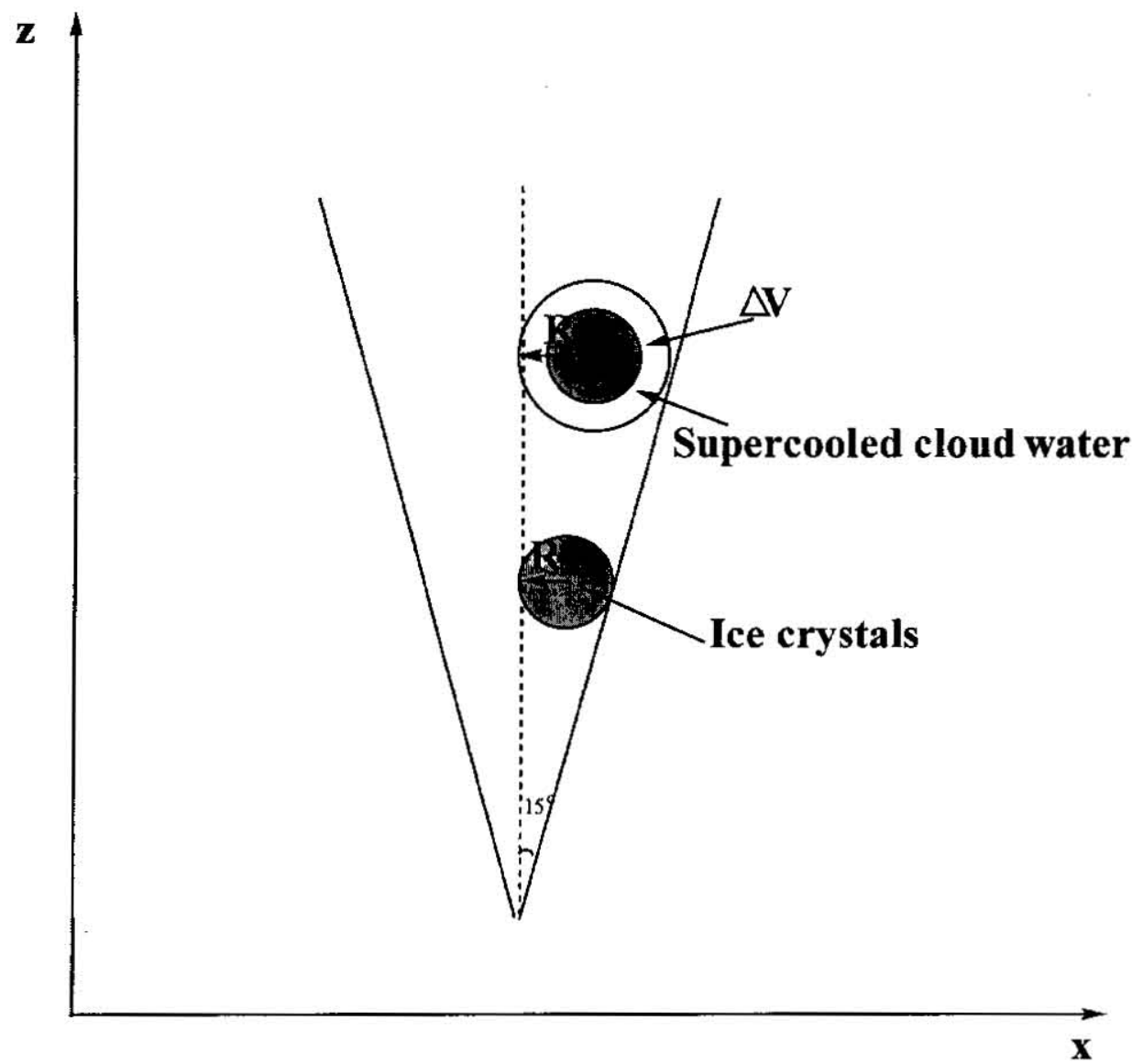

Fig. 1. The process of Roll-up Expansion of Twin Horizontal Ice-crystal Thermal(RETHIT) in liquid carbon dioxide seeding.

\section{Microphysics-dynamies interaction}

\section{a. Buoyarecy}

Ice crystal growth following the nucleation leads to generation of the heat of phase change, which is close to the latent heat of fusion under coexistence of the supercooled droplets. The heat of the phase change warms the air, and ice crystals will become a thermal. The buoyancy of the ice thermal, caused by the heating, drives the thermal upwards and updraft forms. As long as ice crystals grow, the heat generation continuously takes place to sustain the buoyancy and updraft.

According to Fukuta (1973), the heating by the phase change due to the freezing of droplets and the vapor phase adjustment (deposition or sublimation) is given as 


$$
\Delta T=\left[\frac{\left(S_{u^{2}}(T)-1\right) R_{d} T^{2}}{\varepsilon L_{d}}-\frac{L_{f} W_{L}}{\rho_{a} C_{p}}\right]\left(1+\frac{c_{p} R_{d} T^{2}}{\varepsilon q_{i s}(T) L_{d}^{2}}\right)^{-1}+\frac{L_{f} W_{L}}{\rho_{a} c_{p}},
$$

where $S_{w i}(T)$ is the ratio of water saturation vapor pressure to ice saturation vapor pressure, $R_{d}$ the specific gas constant of dry air, $T$ the temperature, $\varepsilon$ the ratio of specific gas constant between dry air and water vapor, $L_{d}$ the specific latent heat of deposition, $L_{f}$ the specific latent heat of fusion, $W_{L}$ the liquid water content (per unit volume) befor the phase change, $\rho_{a}$ the air density, $c_{p}$ the specific heat at constant pressure and $q_{i s}$ the ice saturation mixing ratio.

When the ice thermal rises and the supercooled cloud water is entrained, the water vapor transfers from the droplets onto ice crystals. In this model, we use ice pseudoadiabatic lapse rate for computing the heating due to phase change in the thermal and the ice pseudoadiabatic lapse rate, $\Gamma_{i s}$, is given by

$$
\Gamma_{i s}=\Gamma \frac{1+\frac{L_{d} q_{i s}}{R_{d} T}}{1+\frac{L_{d}^{2} \varepsilon q_{i s}}{R_{d} c_{p} T^{2}}} .
$$

\section{b. Vertical velocity of thermal}

The motion of ice thermal is essentially the same as that of cumulus convection, except that the thermal is in the form of a horizontal line at the beginning of seeding and it occurs inside the cloud. As the thermal rises, it becomes twin rotating cylinders due to the buoyant force acting at the center instead of toroidal shape of the cumulus convection. The twin cylinders of ice thermal roll up inside out and expand while entraining the surrounding supercooled cloud with the help of generated eddy field and sustaining ice crystal growth for generation of latent heat which, in feedback, powers the roll up expansion. If the cumulus model is applied, the semi-vertical angle of the expansion should be about $15^{\circ} \mathrm{C}$ (Emanuel 1994).

In this model, the vertical velocity of thermal is taken according to the bubble theory as

$$
w_{B}=c(g B(2 R))^{a .5},
$$

where $\mathrm{B}=\left(T_{p}-T_{c}\right) / T_{c}$ is the buoyancy factor, $T_{p}$ the ice thermal temperature, $T_{c}$ the ambient cloud temperature, $R$ the radius of a cylinder and $c$ a factor assumed to be 1.2 (Scorer 1957).

\section{c. Terminal velocity of ice crystals}

An ice crystal falls due to the gravitational pull, and the air surrounding the ice crystal exerts resistance to it. When the resistance force balances with the gravitational force, the fall velocity attains a constant value called terminal velocity. For ice crystals, the terminal velocity may be expressed as 


$$
w_{i}={ }_{9}^{2} \underset{\eta}{g \rho_{i} r_{i}^{2}}\left(\operatorname{Re} C_{D} / 24\right),
$$

where $\mathrm{g}$ is the acceleration of gravity, $\rho_{i}$ the density of ice crystal, $\gamma_{i}$ the radius of ice crystal, $\eta$ the dynamic viscosity of air, Re the Reynolds number and $C_{D}$ the drag coefficient. The empirical equation proposed by White (1974) for drag coefficient which agrees well with experimental data for $\operatorname{Re}<5 \times 10^{3}$ is given by

where

$$
C_{i)}=0.25+\frac{24}{\operatorname{Re}}+\frac{6}{1+\sqrt{\operatorname{Re}}},
$$

$$
\mathrm{Re}=\frac{\rho_{a} w_{i}\left(2 r_{i}\right)}{\eta}
$$

Using (7) and (8) in (6), the equation for the terminal velocity of ice crystal may be written as

$$
\begin{aligned}
& 0.25\left(\frac{2 r_{i} \rho_{a}}{\eta}\right)^{1.5} w_{i}^{2.5}+6.25\left(\frac{2 r_{i} \rho_{a}}{\eta}\right) w_{i}^{2}+24\left(\frac{2 r_{i} \rho_{a}}{\eta}\right)^{0.5} w_{i}^{1.5}+24 w_{i}= \\
& -\frac{16 g \rho_{i} r_{i}^{2}}{3 \eta}\left(\frac{2 r_{i} \rho_{a}}{\eta}\right)^{0.5} w_{i}{ }^{0.5}-\frac{16 g \rho_{i} r_{i}^{2}}{3 \eta} .
\end{aligned}
$$

The above equation can be solved by Bisection/Newton-Raphson method and consequently the vertical velocity of ice thermal is given by

$$
w=w_{B}-w_{i} .
$$

The fall velocity of ice crystals $\left(w_{i}\right)$ remains smaller than the updraft velocity of thermal due to buoyancy $\left(w_{B}\right)$, and their separation due to fall occurs when the thermal reaches the top portion of the cloud or even above. Then, the upward motion stops and the ice crystals fallback.

The process shown in Fig. 2 occurs after the RETHIT process in LC seeding. The thermal stops the rotating motion of twin cylinders as it becomes stationary at the top portion of the cloud and flattens. The fall of ice crystals proceeds from the flattened thermal into the underlying supercooled cloud to generate the heat of phase change. The generated heat causes upward motion of air and induces a lateral movement in the top-capped thermal and spreads the ice crystals horizontally. This FILAS effect widens the area of falling ice crystals and, therefore, increases the volume of cloud for treatment and enhances the dynamic effect in the seeding.

\section{d. Action of centrifugal force on ice particles}

When the ice thermal rotates and rises, the centrifugal force acts on ice particles which is given by 


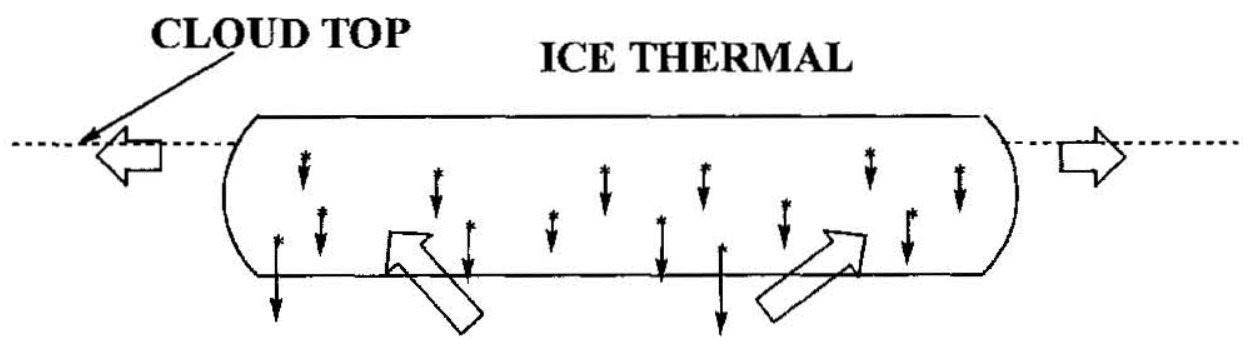

\section{$\widehat{\bigcup \jmath}$ \\ INDUCED UPDRAFT}

Fig. 2. The process of Falling-growth Induced Lateral Air Spreading (FILAS) following RETHIT process in liquid carbon dioxide seeding.

$$
F_{c}=m_{i} \alpha_{c}
$$

where $m_{i}$ is the mass of an ice particle and $a_{c}$ the centrifugal acceleration. The velocity of rotation at one of the cylinders can be expressed by

$$
v_{i}(t)=w(t) R(t)
$$

where $R(t)$ represents the radius of one of the cylinder, and $w(t)$ the angular velocity of thermal, is given by

$$
w(t)=\frac{v_{t}(t)_{R(t)=R_{\max }(t)}^{!}}{R_{\max }(t)},
$$

where $v_{t}(t)=w_{s}(t)$ is the velocity of rotation of thermal at the maximum radius of thermal.

From the balance between the centrifugal force and the viscous resistance force, the radial velocity of ice crystal thermal may be written as

$$
w_{i R}(t)=\frac{2 \rho_{i} R(t) w(t) \underline{t} r_{i}^{2}}{9 \eta}
$$

The horizontal and vertical components of the velocity are written respectively as

and

$$
u(t)=v_{t}(t) \sin \theta+w_{i R}(t) \cos \theta,
$$

$$
w(t)=-v_{t}(t) \cos \theta+w_{i R}(t) \sin \theta+w_{B}(t)-w_{i}(t),
$$




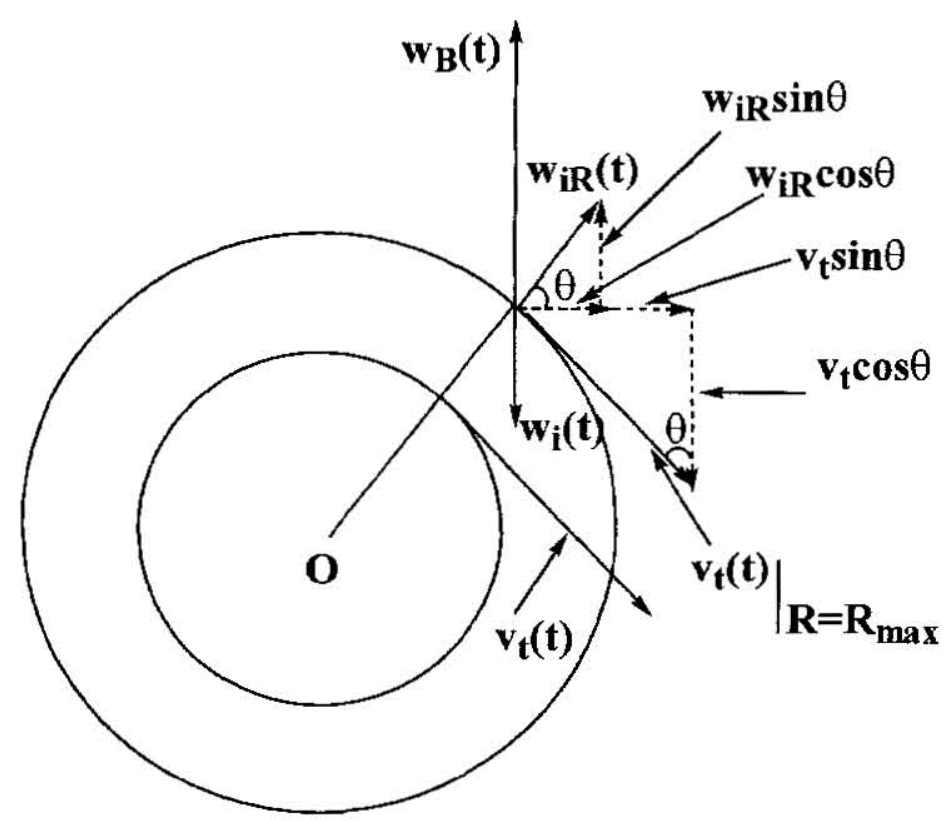

Fig. 3. Vector components of $\mathrm{w}_{\mathrm{B}}, \mathrm{w}_{\mathrm{i}}, \mathrm{w}_{\mathrm{k}}$ and $\mathrm{v}$ in the right-hand side cylinder of the thermal.

where $w_{i R}(t)$ is the radial velocity and $w_{i}(t)$ the fall velocity of ice crystals which are illustrated in Fig. 3.

\section{Numerical procedure}

The model is time-dependent, two dimensional, and covers a region $4 \mathrm{~km} \times 6 \mathrm{~km}$ in the $x$ and $z$ directions. The space increment is $50 \mathrm{~m}$ in the $x$ and $z$ directions and the time increment is $5 \mathrm{~s}$. We assume that a supercooled cumulus cloud has been already formed and liquid water content as constant with height $\left(0.5 \mathrm{~g} / \mathrm{m}^{3}\right)$. The ice crystals are assumed to be spherical in shape with a density of $\rho_{i}=0.917 \mathrm{~g} / \mathrm{cm}^{3}$. The total number of ice crystals in the expanding and rising thermal is constant.

The actual calculations proceed in the following manner:

1. $w_{B}$ is calculated from the known $B$ and $R$ using (5).

2. Vertical velocity of thermal is calculated from the known $w_{i}$ using (10).

3. New position and radius of thermal at the next step is determined.

4. $\Delta m_{i}$ and ice crystal number concentration at the next step are calculated from the known $\Delta \mathrm{V}$.

This completes one cycle of time integration. 


\section{Initial conditions}

It is assumed that the embedded cumulus cloud has $3 \mathrm{~km}$ thickness with the height of the cloud base at $2 \mathrm{~km}$ and the temperature at $0^{\circ} \mathrm{C}$. Temperature in the cloud decreases with height along the moist adiabat.

The temperature at the ground is taken as $20^{\circ} \mathrm{C}$, and it decreases with dry adiabatic lapse rate $(\Gamma)$ until $2 \mathrm{~km}$ level (cloud base) is reached. Horizontal penetration seeding using $\mathrm{LC}$ is carried out at $-1^{\circ} \mathrm{C}$ with a seeding rate of $\mathrm{P}=10 \mathrm{~g} / \mathrm{s}$.

\section{RESULTS AND DISCUSSIONS}

After LC seeding, the formed ice thermal expands its volume, entraining and consuming the ambient supercooled cloud, and reaches an altitude of about $5610 \mathrm{~m}$ at $17.3 \mathrm{~min}$ as shown in Fig. 4, where the radius of the ice thermal (diameter of the cylinder) is $904 m$ as shown in Fig. 5 , the temperature approximately $-24^{\circ} \mathrm{C}$, and air density $0.81 \mathrm{~kg} / \mathrm{m}^{3}$. The total number of ice crystals generated by $1 \mathrm{~m}$ of LC seeding is about $10^{12}$ which doesn't change with time, but number concentration of ice crystal decreases with time. By expansion during the lifting, the number concentration of ice crystals at the cloud top is about $7 \times 10^{7} \mathrm{~m}^{-3}$ as shown in Fig. 6 . Vertical velocity of the ice thermal increases with time due to buoyant force increase, as shown in Figs. 7 and 8 . At $15.3 \mathrm{~min}, w_{\mathrm{t}}$ reaches its maximum value, $7.4 \mathrm{~m} / \mathrm{s}$, then decreases very rapidly with time. Also, the temperature difference between the ice thermal and the supercooled cloud shows a maximum value of approximately $1.3^{\circ} \mathrm{C}$ where the cloud temperature is about

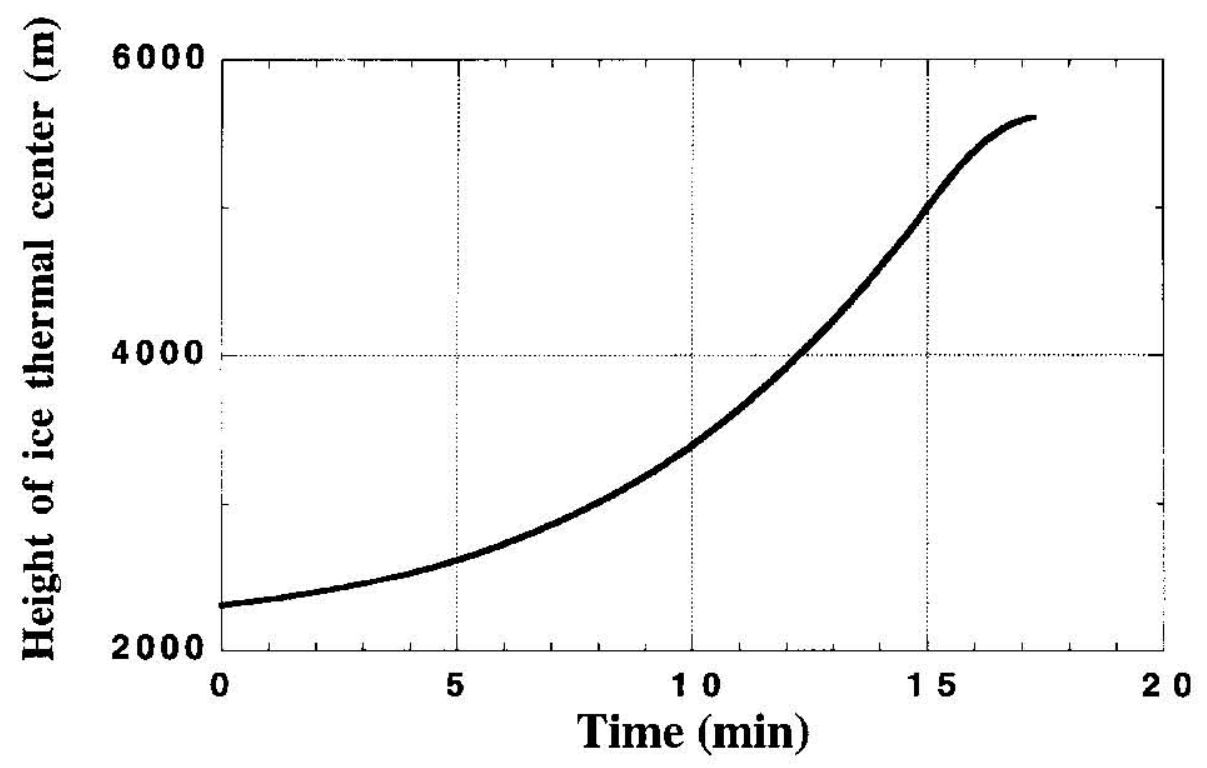

Fig. 4. Time variation of the height of thermal. At $5610 \mathrm{~m}$, the temperature is al $-24^{\circ} \mathrm{C}$ with the air density of about $0.80 \mathrm{~kg} / \mathrm{m}$. 


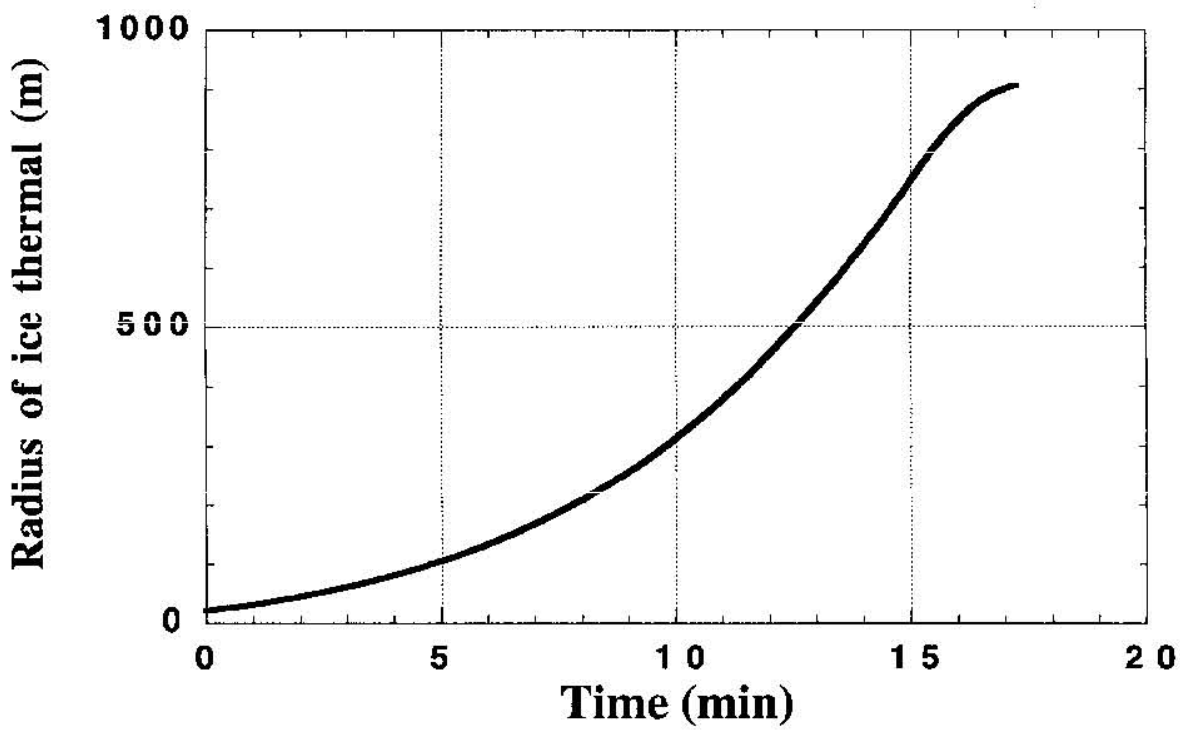

Fig. 5. Time variation of the radius of thermal. At $5610 \mathrm{~m}$, temperature is at $-24^{\circ} \mathrm{C}$ with the air density of about $0.80 \mathrm{~kg} / \mathrm{m}^{\prime}$.

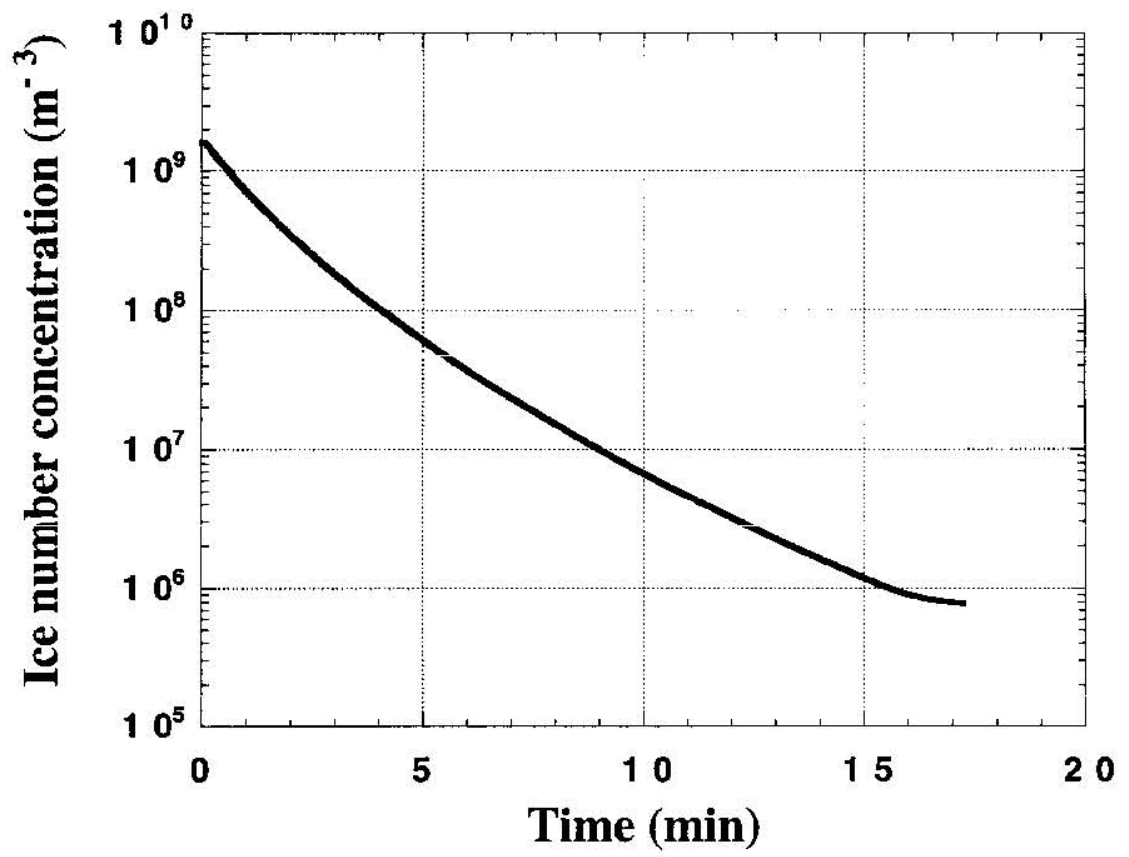

Fig. 6. Time variation of the ice number concentration. Seeding rate is $10 \mathrm{~g} / \mathrm{s}$ and the total number of ice crystals in the thermal is $10^{12} / \mathrm{mr}$. 


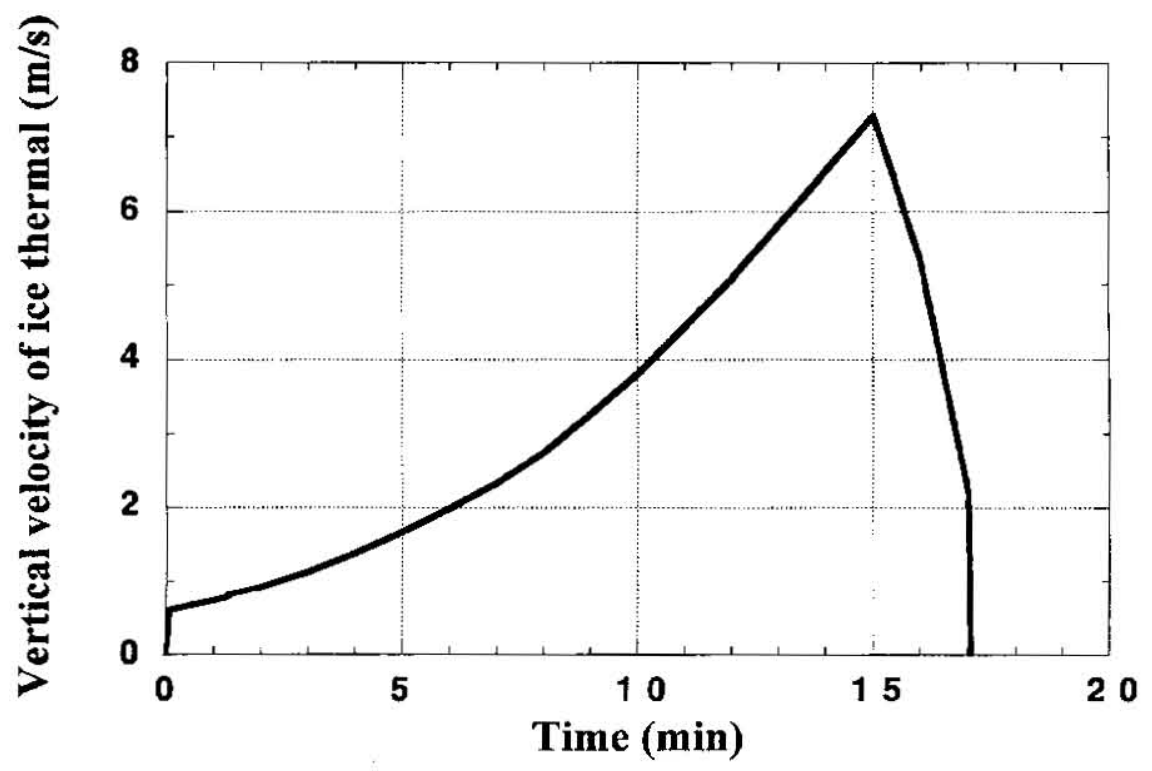

Fig. 7. Time variation of the vertical velocity of thermal due to buoyancy $\left(w_{H}\right)$. At maximum, the cloud temperature is at $-21.5^{\circ} \mathrm{C}$ and the thermal temperature is $-20.2^{\circ} \mathrm{C}$.

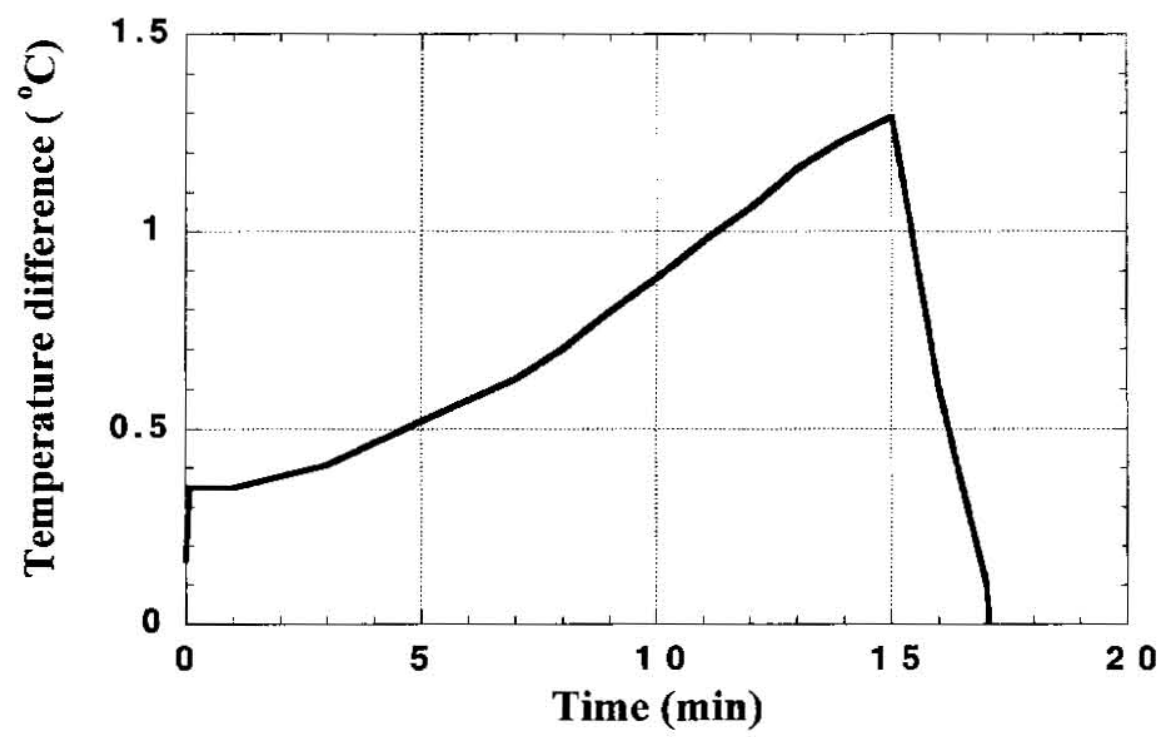

Fig. 8. Time variation of the temperature difference between the cloud ambient and the thermal. At maximum, the cloud temperature is at $-21.5^{\circ} \mathrm{C}$ and the thermal temperature is at $-20.2^{\circ} \mathrm{C}$. 


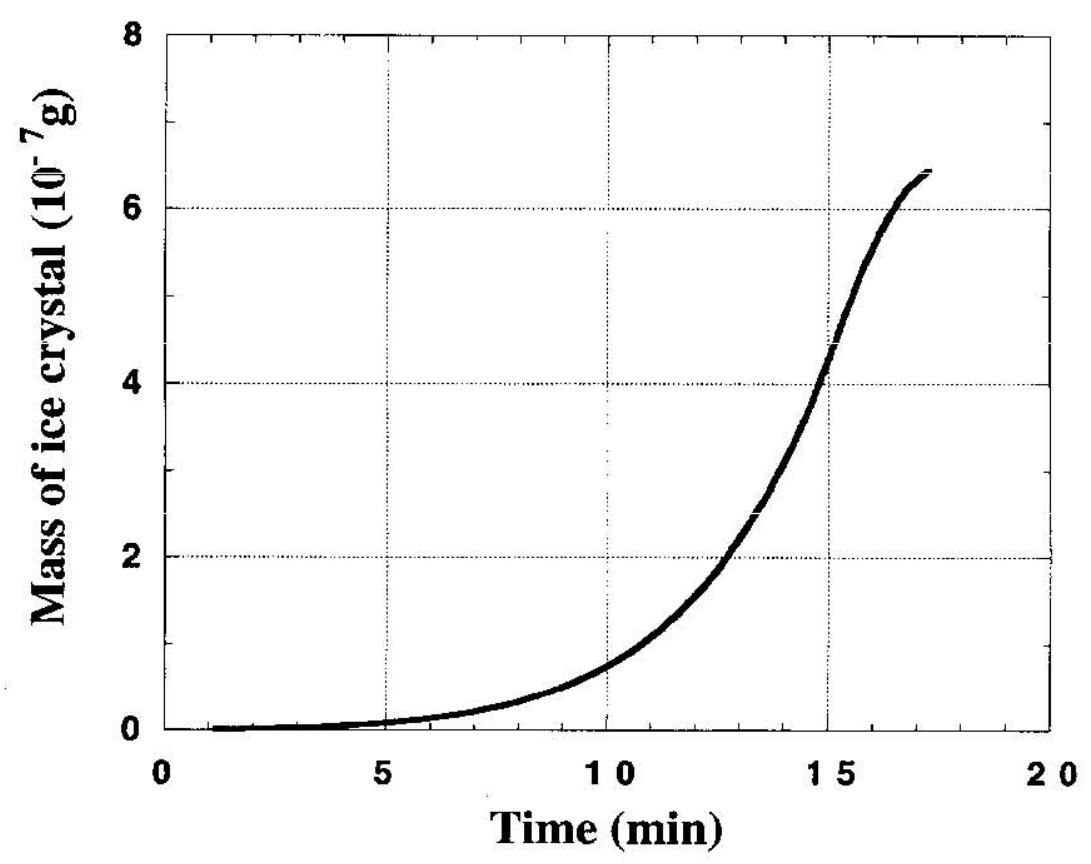

Fig. 9. Time variation of mass of ice crystals. Density of the ice crystal is $0.917 \mathrm{~g} / \mathrm{cm}^{3}$ and the liquid water content of cloud is $0.5 \mathrm{~g} / \mathrm{m}^{4}$

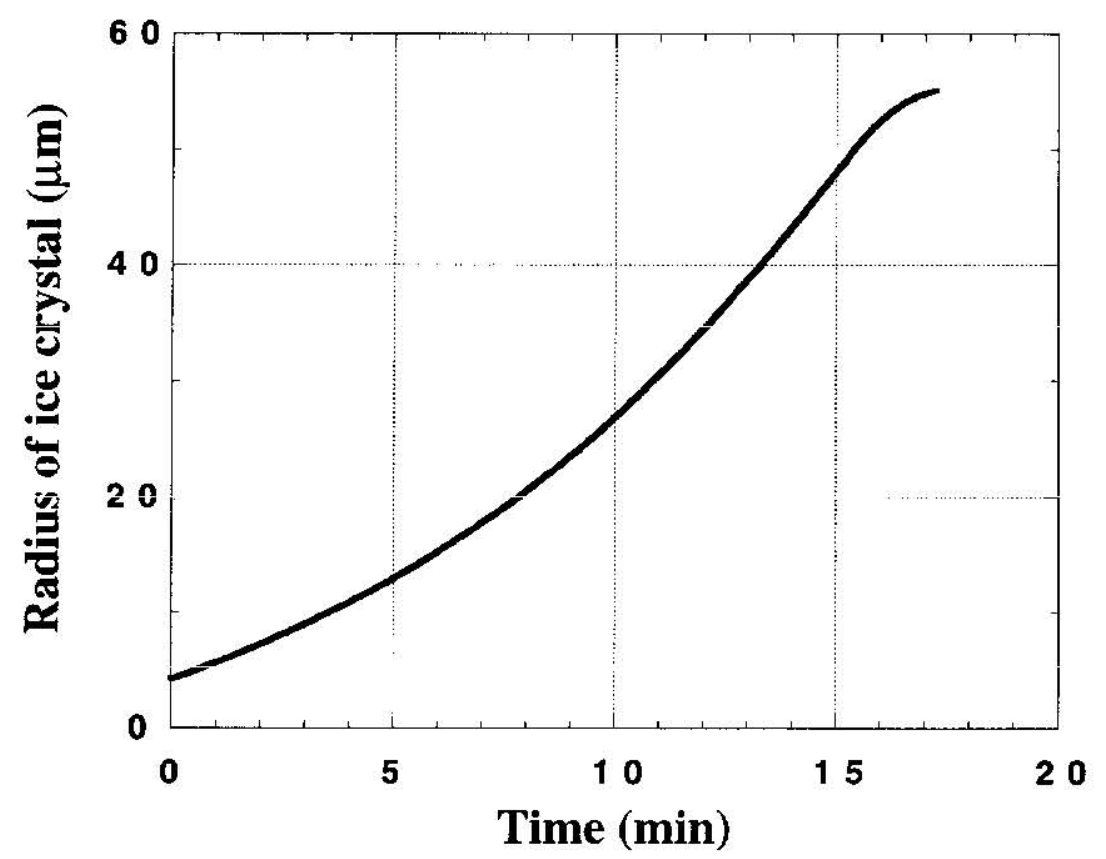

Fig. 10. Time variation of the radius of ice crystals. Density of the ice crystal is $0.917 \mathrm{~g} / \mathrm{cm}^{3}$. 


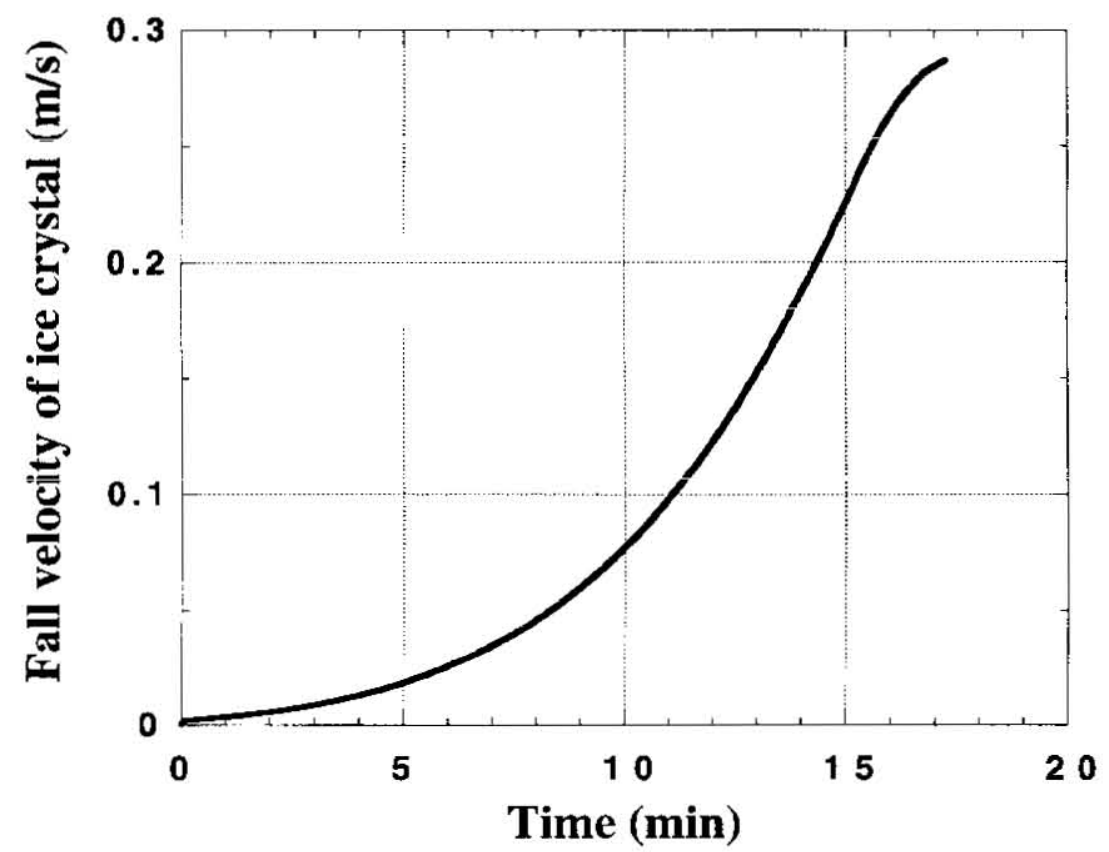

Fig. 11. Time variation of the fall velocity of ice crystal $\left(w_{i}\right)$. Density of the ice crystal is $0.917 \mathrm{~g} / \mathrm{cm}^{4}$ and liquid water content of the cloud is $0.5 \mathrm{~g} / \mathrm{m}^{\prime}$.

$-21.5^{\circ} \mathrm{C}$, air density about $0.83 \mathrm{~kg} / \mathrm{m}^{3}$ and the thermal temperature $-20.2^{\circ} \mathrm{C}$. The ice crystals grow by consuming the liquid water content of cloud entrained into the thermal and the ice crystal mass attains its maximum value, about $6 \times 10^{-} \mathrm{g}$, at somewhat above the cloud top as shown in Fig. 9. The size of ice crystals becomes large enough to fall where the ice crystal radius attains its maximum value, about $55 \mu \mathrm{m}$, as shown in Fig. 10 and the gained fall velocity is about $0.28 \mathrm{~m} / \mathrm{s}$ a little above the cloud top as shown in Fig. 11. Considering the fall velocity of ice crystals, the maximum of ice thermal velocity is about $7.2 \mathrm{~m} / \mathrm{s}$.

As ice thermal moves upward while rotating, the centrifugal force applies to the ice crystals. The movement of ice crystals inside the thermals considering centrifugal force is shown in Figs. 12 through 14. The characteristics of thermal in Figs. 12 through 14 are shown in Table.1. Figure 12 denotes the movement of ice crystals in the thermal at 10 min after LC seeding. The ice thermal radius (diameter of one of the cylinders) is about $312 \mathrm{~m}$ at $3393 \mathrm{~m}$ where the temperature of the thermal is $-7.5^{\circ} \mathrm{C}$ and the cloud temperature $-8.4^{\circ} \mathrm{C}$ with air density about $0.94 \mathrm{~kg} / \mathrm{m}^{\prime}$. The velocity of ice thermal is maximum at the center of the thermal. It is about $7.4 \mathrm{~m} / \mathrm{s}\left(2 w_{B}\right)$, and zero in the right and left sides (outside) of a thermal. The movement of ice crystals in the thermal at 13 min after LC seeding is shown in Fig. 13 where the radius of the thermal is about $540 \mathrm{~m}$ at $4234 \mathrm{~m}$ altitude, the temperature of the thermal $-13.1^{\circ} \mathrm{C}$ and the cloud temperature $-14.3^{\circ} \mathrm{C}$ with air density about $0.89 \mathrm{~kg} / \mathrm{m}^{3}$. Velocity at the center of the 
thermal is $11.2 \mathrm{~m} / \mathrm{s}\left(2 w_{B}\right)$. Figure 14 is the sarne as Figs. 12 and 13 , except at 16 min.

The above described results of the numerical model need some more detailed calculation for the microphysical process. In the model, total liquid water entrained evaporates to absorb the latent heat of condensation and then deposits on the ice crystals to release latent heat of deposition and the net cffect is determined by ice saturation

Table 1. Characteristics of ice thermal after 10,13,16 min after liquid carbon dioxide seeding

\begin{tabular}{ccccc}
\hline Figures & Time(min) & Height(m) & Radius(m) & Velocity(m/s) \\
\hline 11 & 10 & 3393 & 312 & 7.4 \\
12 & 13 & 4234 & 540 & 11.2 \\
13 & 16 & 5210 & 802 & 13.2 \\
\hline
\end{tabular}

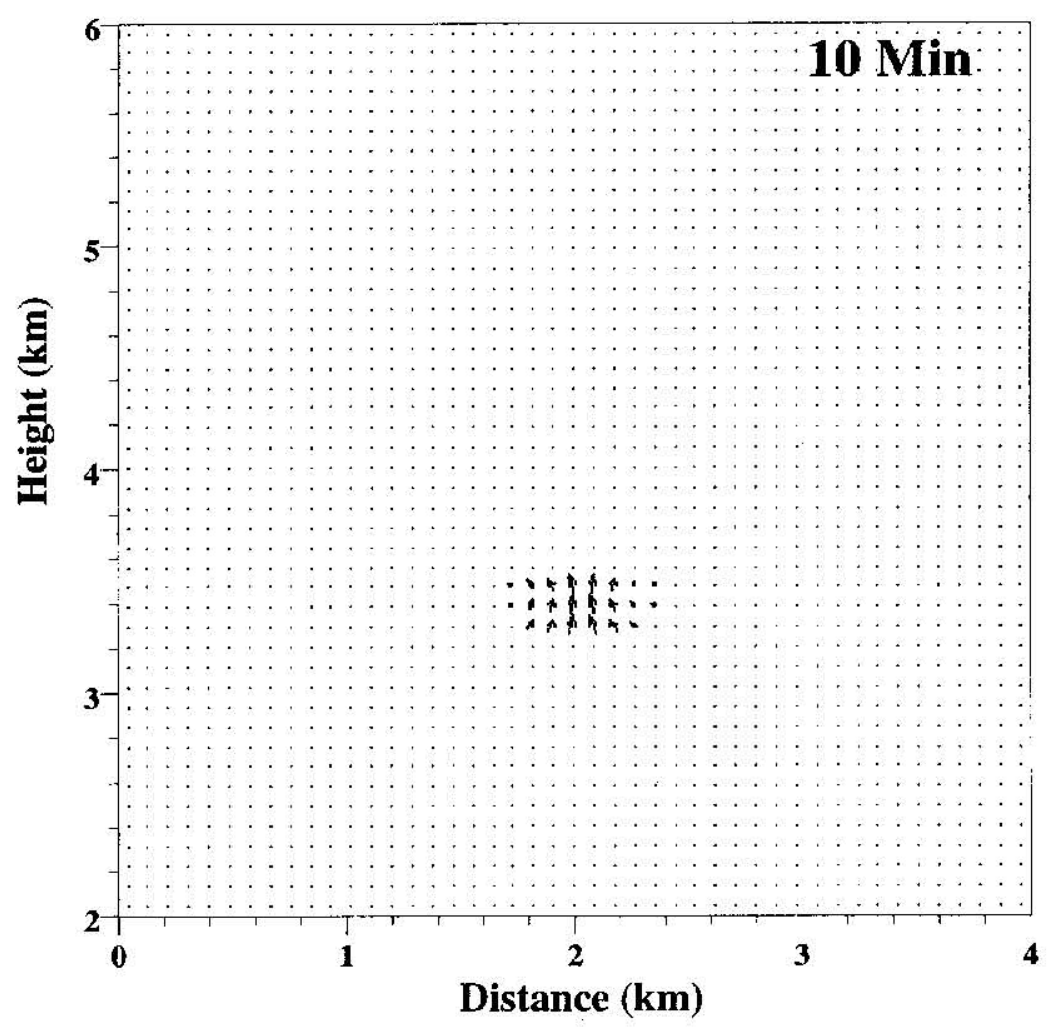

Fig. 12. The stream of thermal at $10 \mathrm{~min}$. 


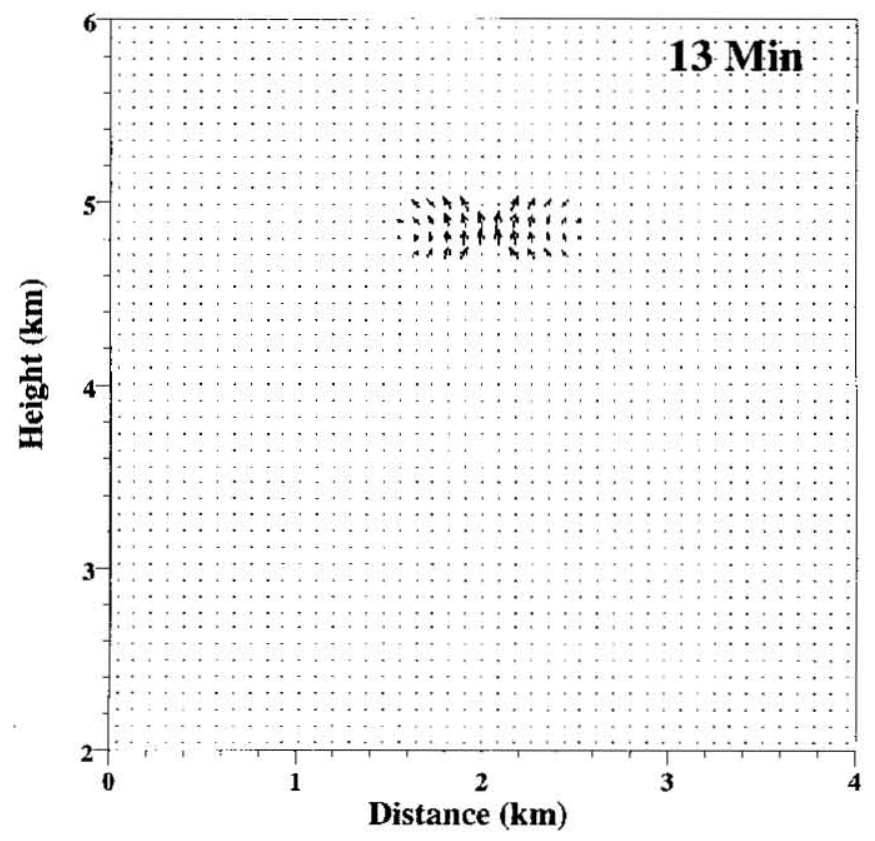

Fig. 13. The stream of thermal at 13 min.

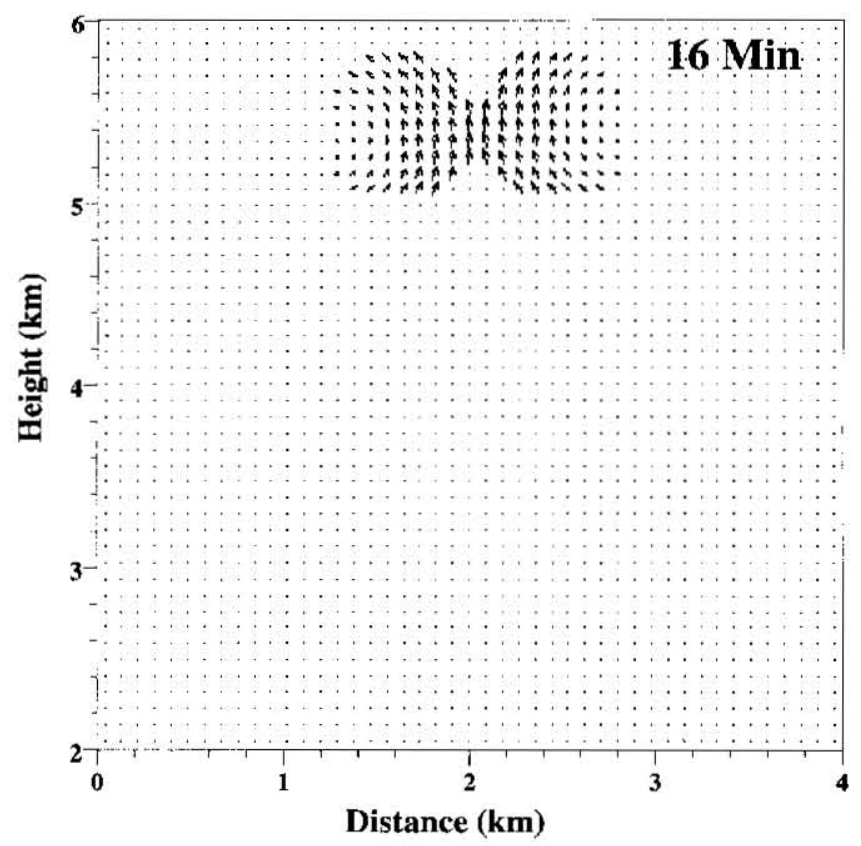

Fig. 14. The stream of thermal at $16 \mathrm{~min}$. 
lapse rate. In reality, some of the liquid water entrained in the thermal will freeze and generate additional heat that shifts the phase equilibrium. Under a high liquid water content and a high temperature (slight supercooling), the vapor deposition and heat generation indicated by the ice saturation lapse rate will have to be reduced and its exact evaluation requires the thermodynamic solution obtained by Fukuta (1973).

The fall velocity used in the present modeling is for spherical particles and gives relatively large values. After 15 min of growth, it reaches about $23 \mathrm{~cm} / \mathrm{s}$ whereas it is only about $17 \mathrm{~cm} / \mathrm{s}$ after fast free growth at $-15^{\circ} \mathrm{C}$ (Takahashi et al. 1991). The spherical assumption for the crystal shape of the present model is, however, close to the reality, because the aspect ratio of the crystals grown under low supersaturations becomes close to unity, and the supersaturation in the present ice crystal thermal is low due to effective moisture depletion by the growing ice crystals in the thermal.

In regard to the calculation of the mass of ice crystals, the present model assumes a total glaciation, or instantaneous conversion of entrained liquid water, which is valid for the case of high number concentration of ice crystals. For $15 \mathrm{~min}$ of growth, an ice crystal achieves the mass of $4.3 \times 10^{7} \mathrm{~g}$ under ascent. Whereas, according to Takahashi et al. (1991), the experimentally determined mass of ice crystal grown for the same period of time at $-15^{\circ} \mathrm{C}$ being surrounded by supercooled droplets, reaches to about $2 \times 10^{-5} \mathrm{~g}$. If the crystal were assumed to have grown under ascent but being surrounded by the droplets, the supersaturation may be assumed linearly proportional to the time. Then, the mass of the crystal would attain amounts about a half of that under the constant temperatures, or $10{ }^{5} \mathrm{~g}$. This is to say that the present modeling gives the means only about $1 / 23$ of the free growth and is consistent with the total glaciation condition taken. Reduction of the seeding rate brings this factor towards unity. However, if the seeding rate were reduced drastically, the competitive growth condition of the ice crystal thermal would soon switch over to the free growth and fail to treat the cloud volume as large as the condition of the present model does.

The assumed seeding rate of $10 \mathrm{gLC} / \mathrm{s}$, which thus resulted in smaller crystals due to their excessive number, may be affected by the ice nucleation rate of LC which is probably a slight overestimation, particularly under engineering inefficiency of actual seeding.

The vertical velocity of the thermal calculated by the bubble theory may be too high. As some of the buoyant energy goes to the energy of rotation of thermal, the resultant vertical velocity should be lower compared with the above results.

\section{ACKNOWLEDGEMENTS}

It is a great pleasure for one of the authors (S. Javanmard) to acknowledge the Ministry of Education, Science, Sports and Culture (Monbusho) of Japan and also the Iranian Meteorological Organization, as well as the Ministry of Culture and Higher Education of the Islamic Republic of Iran, for the financial support during her Ph.D. course in Kyushu University, Fukuoka, Japan. 


\section{REFERENCES}

Cooper, C. F., and W. C. Jolly: 1970 Ecological effects of silver iodide arld other weather modification agents. A review. Water resources Res., 6: 88-89.

Emanuel, K. A., 1994 Atmospheric Convection. Oxford Univ. Press, N. Y., 580 pp.

Fukuta, N., 1963 Ice nucleation by metadehyde. Nature, 19: 475-476.

\section{$454-456$.}

1966 experimental studies of organic ice nuclei. J. Atmos. Sci., 23: 191-196.

1973 Thermodynamics of cloud glaciation. J. Atmos. Sci., 30: 1645-1649.

1987 The surface temperature of dry ice, solid $\mathrm{CO}_{2} . J$. Wea. Mod., 19: 99-101.

1988 The maximum rate of homogeneous ice nucleation in air by cooling. Proceedings $12^{\text {th }}$

Intl. Conf. on Nucleation and Atroos. Aerosols., Vienna, Springer-Verlag, 504-507.

1996a Project Mountain Valley Sunshine-Progress in science and tcchnology. J. Appl.

Meteor., 35: 1483-1493.

$1996 \mathrm{~b}$ Low level penetration seeding with homogeneous icc nucleant for optimization of the induced microphysics-dynamics intcractions. Proceedings $13^{\text {th }}$ Conf on Planned and Inadvert. Wea. Mod., Allanta, 164-171.

: 1998 Cloud sceding clears the air. Physics World., 11:25-26.

W. A. Schmeling. and L. F. Evans, 1971 Experimental determination of the ice nucleation by falling dry ice pellets. J. Appl. Meteor., 10: 1174-1179.

Garvey: D. M., 1975 Testing of cloud seeding materials at the Cloud Simulation and Acrosol Laboratory, 1971-1973, J. Appl. Meteor., 14: 883-890.

Klein, D. A., 1978 The Environmental Impacts of Artificial Ice Nucleating Agents, Dowden, Hutchirisun and Rose, Stroudburg, Ponnsylvania, $257 \mathrm{pp}$.

Plooster, M. N., and N. Fukuta, 1975 A numerical model of precipitation from seeded and unsecded orographic clouds. J. Appl. Meteor, 14: 859-867.

Schacfer, V. J., 1946 The production of ice crystals in a cloud of supercooled water droplets. Science. $104: 457-459$.

Schlaller, R. C., and N. Fukuta, 1979 Ice nucleation by aerosol particles: Experimental studies using a wedge-shaped ice thermal diffusion chamber. J. Atmos. Sci., 36: 1788-1802.

Scorer, R. S. 1957 Experiments on convection of isolated masses of buoyant fluid. J. Fluid Mech., 2: $583-596$.

Takahashi, T., T. Endoh, G. wakahama and N. Fukuta, 1991 Vapor diffusional growth of frec-falling snow crystals between -3 and $-23^{\circ} \mathrm{C}$. J. Meleor: Soc. Japan, 69: 15-30.

Vonnegut, B., 1974 Nucleation of ice formation by silver iodide. J. Appl. Phys., 18: 593-595.

White, F. M., 1974 Viscous Fluid Flow. MacGraw Hill, 725 pp. 